\title{
Response of the National Biosurveillance Integration Center to the Emergence of Porcine Epidemic Diarrhea Virus in the United States
}

\author{
Yandace K. Brown* and Tyann Blessington \\ National Biosurveillance Integration Center, Office of Health Affairs, Department of Homeland Security, Washington D.C., DC, USA
}

\section{Objective}

NBIC utilized information from various sources to communicate pertinent information on the emergence of Porcine Epidemic Diarrhea Virus (PEDV) in the United States in written products to be distributed to its Federal partners.

\section{Introduction}

The United States Department of Agriculture (USDA) Animal and Plant Inspection Service (APHIS) announced the first diagnosed case of PEDV in U.S. swine in Iowa on May 17, 2013. PEDV subsequently spread rapidly among the domestic swine herds throughout the United States. As of August 20, 2014, PEDV had been detected in 30 U.S. states affecting more than seven million pigs since the epidemic began. In the first year of emergence, PEDV reporting was voluntary, but on June 5, 2014, the Secretary of Agriculture issued a Federal Order that all cases of swine enteric coronavirus diseases (SECDs), including PEDV, were required to be reported to the USDA APHIS.

PEDV only affects swine and is not a public health concern. However, NBIC was interested in reporting on this disease due to the economic impact. Swine and pork products are valuable contributors to the domestic and international markets.

\section{Methods}

On June, 4 2013, PEDV first appeared as an item on the National Biosurveillance Integration System (NBIS) Reporting List. The daily NBIS Reporting List is distributed to Federal as well as state and local partners that contains brief summaries of the events being monitored. Information regarding the progression and milestones of PEDV emergence, research, and other developments were obtained from open source media reports and updates collected on the American Association of Swine Veterinarians (AASV) website. Recognizing USDA APHIS as the lead Federal agency and one of its partners, NBIC coordinated a brief conference call with representatives from USDA APHIS on December 16, 2013 to discuss the disease emergence and current response to the disease event. As PEDV continued to spread within and between U.S. states, the event was elevated to the level of a biosurveillance event report (BER), which was first written on January 24, 2014.

\section{Results}

In the beginning, experts had predicted that the economic impact would be rather costly considering the importance of the pork industry to agriculture and the observed high mortality of the virus. While the swine industry and pork prices are still suffering an impact due to PEDV, the economic decline is not as drastic as predicted. Over the course of the virus' emergence, six countries have imposed import restriction on U.S. pigs and pork products. At least two of those countries, China and Japan, have since lifted their restrictions after the Federal Order was put into place.

The epidemic peaked in the winter of 2013 - 2014. Open source reports suggest that this pattern may possibly be due to a harsh winter contributing to viral spread in frozen manure on trucks used to haul pigs between farms and other production locations. Animal health experts anticipate PEDV in the U.S. to increase again in the fall of 2014 as temperatures grow cooler and outdoor conditions become damper. Though seasonality should be considered, the lack of a coordinated response from a single authoritative agency may have been an important factor in the rapid spread of the virus across the country. Industry organizations and academic institutions exerted a strong response to the emergence of PEDV; however, there was no single agency ensuring coordination between or within states.

\section{Conclusions}

Without a formal response protocol by a single coordinating body, PEDV spread rapidly between states. Several sources have proclaimed that the emergence and spread of PEDV has awakened the U.S. livestock industry and governments to lapses in current biosecurity systems and the need to increase vigilance to avoid having other dangerous foreign animal diseases (FADs) enter the country. Though APHIS has been actively involved from the beginning of the PEDV emergence, the Federal Order has marked a more formalized and coordinated response, which NBIC predicts will exert greater control over the epidemic despite the predicted viral surge in the colder months.

NBIC is mandated to serve as a Federal coordinating center and does not take the lead on any one event. Events such as the emergence of PEDV present opportunities for collaboration with Federal partners.

\section{Keywords}

PEDV; swine; coordination; biosecurity

\section{*Yandace K. Brown}

E-mail: Yandace.Brown@hq.dhs.gov 